\title{
Comparison of Perfusion, Diffusion, and MR Spectroscopy between Low-Grade Enhancing Pilocytic Astrocytomas and High-Grade Astrocytomas
}

M. de Fatima Vasco Aragao, M. Law, D. Batista de Almeida, G. Fatterpekar, B. Delman, A.S. Bader, M. Pelaez, M. Fowkes, R. Vieira de Mello, and M. Moraes Valenca

\begin{abstract}
BACKGROUND AND PURPOSE: The differentiation of pilocytic astrocytomas and high-grade astrocytomas is sometimes difficult. There are limited comparisons in the literature of the advanced MR imaging findings of pilocytic astrocytomas versus high-grade astrocytomas. The purpose of this study was to assess the MR imaging, PWI, DWI, and MR spectroscopy characteristics of pilocytic astrocytomas compared with high-grade astrocytomas.

MATERIALS AND METHODS: Sixteen patients with pilocytic astrocytomas and 22 patients with high-grade astrocytomas (8-66 years of age; mean, $36 \pm 17$ years) were evaluated by using a 1.5T MR imaging unit. MR imaging, PWI, DWI, and MR spectroscopy were used to determine the differences between pilocytic astrocytomas and high-grade astrocytomas. The sensitivity, specificity, and the area under the receiver operating characteristic curve of all analyzed parameters at respective cutoff values were determined.

RESULTS: The relative cerebral blood volume values were significantly lower in pilocytic astrocytomas compared with the high-grade astrocytomas $(1.4 \pm 0.9$ versus $3.3 \pm 1.4 ; P=.0008)$. The ADC values were significantly higher in pilocytic astrocytomas compared with high-grade astrocytomas $\left(1.5 \times 10^{-3} \pm 0.4\right.$ versus $\left.1.2 \times 10^{-3} \pm 0.3 ; P=.01\right)$. The lipid-lactate in tumor/creatine in tumor ratios were significantly lower in pilocytic astrocytomas compared with high-grade astrocytomas $(8.3 \pm 11.2$ versus $43.3 \pm 59.2 ; P=.03$ ). The threshold values $\geq 1.33$ for relative cerebral blood volume provide sensitivity, specificity, positive predictive values, and negative predictive values of $100 \%, 67 \%, 87 \%$, and $100 \%$, respectively, for differentiating high-grade astrocytomas from pilocytic astrocytomas. The optimal threshold values were $\leq 1.60$ for $A D C, \geq 7.06$ for lipid-lactate in tumor/creatine in tumor, and $\geq 2.11$ for lipid-lactate in tumor/lipid-lactate in normal contralateral tissue.
\end{abstract}

CONCLUSIONS: Lower relative cerebral blood volume and higher ADC values favor a diagnosis of pilocytic astrocytoma, while higher lipid-lactate in tumor/creatine in tumor ratios plus necrosis favor a diagnosis of high-grade astrocytomas.

ABBREVIATIONS: GBM = glioblastoma multiforme; $\mathrm{HGA}=$ high-grade astrocytoma; $\mathrm{HGG}=$ high-grade glioma; $\mathrm{LGG}=$ low-grade glioma; Lip-Lac = lipid-lactate; $\mathrm{n}=$ normal contralateral; $\mathrm{PA}=$ pilocytic astrocytoma; $\mathrm{rCBV}=$ relative cerebral blood volume; $\mathrm{ROC}=$ receiver operating characteristic analysis curve; tu $=$ tumor; WHO $=$ World Health Organization

$\mathbf{P}$ ilocytic astrocytoma (PA) is the most common pediatric central nervous system glioma and one of the most common pediatric cerebellar tumors. This tumor occurs most frequently in

Received July 22, 2013; accepted after revision January 16, 2014.

From the Departments of Radiology (M.d.F.V.A., D.B.d.A., B.D., A.S.B., M.P.) and Pathology (M.F.), Mount Sinai School of Medicine, New York, New York; Centro Diagnóstico Multimagem (M.d.F.V.A.), Recife, Brazil; Department of Neuropsychiatry and Behavioral Studies (M.d.F.V.A., M.M.V.), Federal University of Pernambuco, Rec ife, Brazil; Department of Radiology (M.L.), University of Southern California, Los Angeles, California; Department of Radiology (G.F.), New York University Langone Medical Center, New York, New York; and Department of Pathology (R.V.d.M.), Federal University of Pernambuco, Recife, Brazil.

Please address correspondence to Maria de Fatima Vasco Aragao, MD, PhD, Multimagem, Rue Frei Matias Tevis, 194 - Ilha do Leite, Recife, Brazil; and Department of Neuropsychiatry and Behavioral Studies, Federal University of Pernambuco, Recife, Brazil-PE 50070-450; Estrada das Ubaias 332 apt 1201, Casa Forte, Recife-PE, Brazil, CEP-52061-080; e-mail: fatima.vascoaragao@gmail.com

- Indicates open access to non-subscribers at www.ajnr.org

http://dx.doi.org/10.3174/ajnr.A3905 the first 2 decades of life, ${ }^{1}$ composing up to $25 \%$ of brain tumors in pediatric neurosurgical practices, ${ }^{2}$ and it only rarely occurs in adults. $^{2}$ The incidence is approximately 4.8 cases per million people per year, ${ }^{3}$ with $2.3 \%-6 \%$ of all brain tumors classified as PAs. ${ }^{3,4}$ PAs are usually clinically benign and are classified as grade I by the World Health Organization (WHO). ${ }^{4}$ They are potentially curable by surgery and are associated with a longer survival. ${ }^{1,5}$ Very rarely, a PA may undergo spontaneous malignant transformation and become an anaplastic astrocytoma. ${ }^{6}$

A PA typically appears on MR imaging as a large cystic mass with a mural nodule ${ }^{1,5}$; however, this pattern can also occur in a hemangioblastoma of the posterior fossa. ${ }^{7} \mathrm{MR}$ imaging features of PAs can also be seen in high-grade gliomas (HGGs) and metastases, ${ }^{7}$ particularly when a PA appears as a solidly enhancing mass. Moreover, despite their benign biologic behavior, PAs may be confused with malignant tumors and resemble high-grade astro- 
cytomas (HGAs) on both histopathology and during a neuroimaging evaluation, making the diagnosis difficult. ${ }^{1,4}$ This confusion can occur because PAs present a variety of histologic patterns and may have markedly hyalinized and glomeruloid vessels, accompanied by extensive nuclear pleomorphism, which can sometimes make classification a challenge. ${ }^{1,4}$

Given that differentiation of PAs and HGAs is sometimes challenging to both the neuroradiologist and the pathologist, advanced MR imaging techniques that add functional information to the anatomic MR imaging can be helpful in the characterization and grading of astrocytomas. ${ }^{8}$ Although both PA and HGA belong to the same family of gliomas, a great amount of evidence suggests that PAs present histologic aspects (cellularity, vascularity, cystic formation, necrosis) very distinct from those found in HGAs. Thus, the use of these advanced MR imaging techniques may better evaluate and distinguish the anatomic and functional differences occurring between PAs and HGAs. These include the following: diffusion-weighted imaging (DWI), which evaluates the microstructure and cellularity of brain tissue by analyzing the motion of the water in the tissue ${ }^{9}$; perfusion-weighted imaging (PWI), which evaluates the microvasculature by using relative cerebral blood volume ( $\mathrm{rCBV})^{9,10}$; and MR spectroscopy, which provides metabolic and histologic marker information about the brain or neoplastic tissue. ${ }^{9-11}$

There are only a few small series assessing the advanced MR imaging features of PAs, particularly when evaluating neovascularity on $\mathrm{PWI}^{7,12}$ and their differentiation from HGAs. ${ }^{12}$

Our hypothesis is that in comparison with HGAs, PAs present lower cellularity, less vascularity, and a lower metabolism, and each one of these characteristics may be detected with the use of DWI, PWI, and MR spectroscopy, respectively. Therefore, advanced MR imaging techniques should demonstrate findings that may help in the differentiation of PAs from HGAs. The purpose of this study was to assess MR imaging, DWI, PWI, and MR spectroscopy characteristics of PAs compared with HGAs.

\section{MATERIALS AND METHODS Patients and Histopathologic Analysis}

MR imaging studies of 38 patients with astrocytomas were reviewed (16 patients with PAs and 22 patients with HGAs). MR imaging examinations were performed from April 2002 to April 2008. Patient ages ranged from 8 to 66 years, with a mean of $36.23 \pm 16.95$ years. There were 23 male and 15 female patients. In this retrospective study, primarily newly diagnosed, untreated astrocytomas (36/38) were included; only 2 PA cases were studied after biopsy.

Histopathologic evaluation was performed by 2 experienced neuropathologists and was based on the WHO classification of astrocytomas $^{13}$ : grade I, PA; grade II, diffuse astrocytoma; grade III, anaplastic astrocytoma; and grade IV, glioblastoma multiforme (GBM). Only grade I (PA) and grade III-IV (HGA) astrocytomas were included in this study. Grade III and IV astrocytomas were allocated in a single group because their aggressive biologic behavior is quite often similar, but this will depend on many factors including genetic profile. All patients had histopathologic confirmation of PAs and HGAs, with specimens obtained by open excisional biopsy or resection. Our institutional review board approved the study.

\section{Imaging Protocol}

Because this was a retrospective study, a full complement of imaging was not available for each patient. Based on the histopathologic criteria for seeking the signs of morphology that express the greatest aggressiveness, we selected the PWI, DWI, and MR spectroscopy measurements that best correlated with the grading of tumors.

Magnetic Resonance Imaging. Imaging was performed with a 1.5T unit (Signa Infinity; GE Healthcare, Milwaukee, Wisconsin). The MR imaging sequences were performed by using sagittal and axial T1-weighted imaging, axial T2, axial FLAIR, and axial T2*weighted imaging. Contrast-enhanced axial reference T1weighted imaging was performed after the perfusion MR imaging data had been obtained. An experienced neuroradiologist reviewed the conventional MR images and evaluated each lesion on the basis of location (supratentorial or infratentorial), tumor characteristics (solid, cystic, or necrotic), and type of enhancement (none, homogeneous, heterogeneous, or ring).

Dynamic Perfusion MR Imaging. PWI was performed in 9 patients with PA and 20 with HGA. Gadopentetate dimeglumine contrast medium (Magnevist; Bayer HealthCare Pharmaceuticals, Leverkusen, Germany) was intravenously administered, with a contrast dose of $0.1 \mathrm{mmol} / \mathrm{Kg}$ of body weight, by a power injector at a rate of approximately $4 \mathrm{~mL} / \mathrm{s}$, followed by a saline bolus (10-20 $\mathrm{mL}$ at approximately $4 \mathrm{~mL} / \mathrm{s}$ ). Nine to 10 sections were selected for PWI through the tumor, by using T2-weighted images as a guide. Spin-echo PWI was performed by using the following parameters: TR/TE, flip angle, FOV, matrix, section thickness, section gap: $1900 / 80 \mathrm{~ms}, 90^{\circ}, 24 \mathrm{~cm}, 128 \times 64$ pixel, $7 \mathrm{~mm}, 1.5 \mathrm{~mm}$. Preload contrast was administered before the PWI acquisition to correct T1-weighted leakage effects that might underestimate rCBV measurement. Data processing was performed by using an Advantage Windows 4.2 workstation with FuncTool as the analytic program (GE Healthcare). After the construction of an $\mathrm{rCBV}$ color map to target the regions of maximal abnormality, measurements at 4 regions of interest were obtained, and the maximum rCBV was recorded. A standardized region of interest measuring approximately $2-4 \mathrm{~mm}^{2}$ was used. CBV measurements were made relative to the normal-appearing contralateral white matter.

Proton MR Spectroscopic Imaging. Multivoxel 2D MR spectroscopy was performed before the administration of gadopentetate dimeglumine in 5 patients with PAs and 21 with HGAs. Using the T2 sequence as reference for voxel placement, a 2D spectroscopy field was prescribed to contain tumor and contralateral supposedly normal brain parenchyma. A multivoxel MR spectroscopy was performed with the point-resolved spectroscopy technique: TR/TE, 1500/35 ms; VOI thickness, $16.5 \mathrm{~mm}$; FOV, $18 \mathrm{~cm}$; coding phase, $16 \times 16$; NEX, 1 ; direction of the frequency, anteroposterior. All spectra were processed by using an Advantage Windows 4.2 workstation with FuncTool as the analytic program. The metabolite peaks were assigned at the following frequencies: choline (Cho) at $3.22 \mathrm{ppm}$, creatine $(\mathrm{Cr})$ at $3.02 \mathrm{ppm}, \mathrm{N}$-acetylaspartate (NAA) at $2.02 \mathrm{ppm}$, lipid-lactate (Lip-Lac) at 0.5-1.5 ppm, and myo-inositol (mIns) at $3.56 \mathrm{ppm}$. The peak area measurements of metabolites were used to calculate the following ratios: minimal tumor $\mathrm{Cr}$ to contralateral normal $\mathrm{Cr}\left(\mathrm{Cr}^{\mathrm{tu}} / \mathrm{Cr}^{\mathrm{n}}\right)$, maximal tumor 
Table 1: The characteristics of PA and HGA on conventional MR imaging

\begin{tabular}{lccccccc} 
& & & & & \multicolumn{2}{c}{ Type of Enhancement } \\
\cline { 6 - 8 } Tumor & Solid & Solid-Cystic & Necrotic & Enhancement & Heterogeneous & Homogeneous & Ringlike \\
\hline PA & $8 / 16(50 \%)$ & $7 / 16(44 \%)$ & $1 / 16(6 \%)$ & $14 / 15(93 \%)$ & $8 / 14(57 \%)$ & $5 / 14(36 \%)$ & $1 / 14(7 \%)$ \\
HGA & $8 / 22(36 \%)$ & $0 / 22(0 \%)$ & $14 / 22(64 \%)$ & $20 / 22(91 \%)$ & $7 / 20(35 \%)$ & $1 / 20(5 \%)$ & $12 / 20(60 \%)$ \\
\hline
\end{tabular}

Cho to contralateral Cho $\left(\mathrm{Cho}^{\mathrm{tu}} / \mathrm{Cho}^{\mathrm{n}}\right)$, minimal mIns to contralateral mIns $\left(\mathrm{mIns}^{\mathrm{tu}} / \mathrm{mIns}^{\mathrm{n}}\right)$, and maximal Lip-Lac to contralateral Lip-Lac (Lip-Lac ${ }^{\text {tu }} / \mathrm{Lip}-\mathrm{Lac}^{\mathrm{n}}$ ). In addition to ratios against normal tissue, the following intrinsic tumor metabolite ratios were also obtained: minimal $\mathrm{NAA}^{\mathrm{tu}} / \mathrm{Cr}^{\mathrm{tu}}$, minimal $\mathrm{mIns}^{\mathrm{tu}} /$ $\mathrm{Cr}^{\mathrm{tu}}$, maximal $\mathrm{Cho}^{\mathrm{tu}} / \mathrm{Cr}^{\mathrm{tu}}$, maximal $\mathrm{Cho}^{\mathrm{tu}} / \mathrm{NAA}^{\mathrm{tu}}$, and maximal $\mathrm{Lip}-\mathrm{Lac}^{\mathrm{tu}} / \mathrm{Cr}^{\mathrm{tu}}$. These MR spectroscopy criteria were selected to best correlate with the grading of the tumors on histopathology. In this way, among all the multivoxel spectroscopy graphs of the tumor, we chose the metabolite that presented the greatest alteration, independent of its position within the tumor (solid or necrotic). The smallest NAA of all the spectroscopy graphics and the greatest Cho were chosen. When the tumor had necrosis, the greatest Lip-Lac peak coincided with the necrotic area.

Diffusion-Weighted Imaging. DWI was performed in 13 patients with PAs and 12 with HGAs. DWI was performed in the transverse plane by using a spin-echo echo-planar imaging sequence with the following parameters: TR/TE, 5000/78.6 ms; diffusion gradient encoding in 3 orthogonal directions; b-value $=1000$ $\mathrm{s} / \mathrm{mm}^{2}$; FOV , $24 \mathrm{~cm}$; matrix size, $96 \times 192$ pixels; section thickness, $5 \mathrm{~mm}$; section gap, $1.5 \mathrm{~mm}$; and NEX, 2. DWI scans were performed before PWI and contrast-enhanced T1-weighted imaging. The minimal apparent diffusion coefficient (ADC) value was chosen in solid and enhanced areas of the tumor to best characterize the maximal cellularity of the tumor.

\section{Statistical Analysis}

All parameters are reported as means \pm SD. For a comparison of the quantitative variances between the PA and HGA groups, we applied the nonparametric Mann-Whitney statistical test because of the lack of normality of the data.

The sensitivity, specificity, positive predictive value, and negative predictive value of each advanced MR imaging technique used in this study were calculated to differentiate PAs from HGAs.

We used a receiver operating characteristic analysis curve (ROC) to decide the cutoff between PAs and HGAs. The cutoff values chosen were those that provided greater sensitivity and specificity jointly (ie, the best combination of the 2 measures).

The analyses were performed in the Statistical Package for the Social Sciences, Version 12.0 (IBM, Armonk, New York). A P value $<.05$ was statistically significant.

\section{RESULTS}

Within the PA group, 10/16 (62\%) patients were younger than 21 years old at presentation. With regard to the location of the tumor, 9/16 (56\%) cases were supratentorial and 7/16 (45\%) were infratentorial. Among the supratentorial tumors, 6/9 (67\%) were hypothalamic-chiasmatic.

Within the HGA group, most of the patients were adults (21/ $22,95 \%)$ and most tumors were supratentorial (21/22, 95\%). The
Table 2: Comparison of the PA and HGA groups with regard to the variables of interest (mean \pm standard deviation)

\begin{tabular}{|c|c|c|c|}
\hline & PA & HGA & $P$ Value \\
\hline rCBV & $1.408 \pm 0.870$ & $3.322 \pm 1.401$ & .0008 \\
\hline $\mathrm{ADC}$ & $1.534 \pm 0.382$ & $1.193 \pm 0.279$ & .0134 \\
\hline $\mathrm{NAA}^{\mathrm{tu}} / \mathrm{Cr}^{\mathrm{tu}}$ & $1.180 \pm 0.708$ & $1.247 \pm 0.681$ & .8457 \\
\hline $\mathrm{Cho}^{\mathrm{tu}} / \mathrm{NAA}^{\mathrm{tu}}$ & $2.020 \pm 1.090$ & $1.417 \pm 0.782$ & .1628 \\
\hline $\mathrm{Cho}^{\mathrm{tu}} / \mathrm{Cr}^{\mathrm{tu}}$ & $3.158 \pm 2.651$ & $2.838 \pm 1.595$ & .6028 \\
\hline $\mathrm{Cho}^{\mathrm{tu}} / \mathrm{Cho}^{\mathrm{n}}$ & $1.390 \pm 0.743$ & $1.963 \pm 2.275$ & .3958 \\
\hline Lip-Lac ${ }^{\text {tu }} / \mathrm{Cr}^{\text {tu }}$ & $8.326 \pm 11.180$ & $43.320 \pm 59.240$ & .0273 \\
\hline $\mathrm{Cr}^{\mathrm{tu}} / \mathrm{Cr}^{\mathrm{n}}$ & $0.464 \pm 0.234$ & $0.495 \pm 0.326$ & .8437 \\
\hline Lip-Lac ${ }^{\text {tu }} /$ Lip-Lac ${ }^{\text {n }}$ & $1.746 \pm 0.918$ & $6.555 \pm 7.480$ & .0618 \\
\hline $\mathrm{mlns} \mathrm{su}^{\mathrm{tu}} / \mathrm{mlns}^{\mathrm{n}}$ & $1.020 \pm 0.419$ & $0.749 \pm 0.733$ & .0961 \\
\hline $\mathrm{mlns} s^{t u} / \mathrm{Cr}^{\mathrm{tu}}$ & $1.924 \pm 0.468$ & $1.607 \pm 0.9953$ & .1264 \\
\hline
\end{tabular}

Note:-ADC measurements are expressed in $\times 10^{-3} \mathrm{~mm}^{2}$ per second.

main characteristics of PA and HGA on conventional MR imaging are shown in Table 1.

The rCBV values were significantly lower in the PA group compared with the HGA group (Table 2 and Fig 1). Only 3/9 $(33 \%)$ PA cases showed elevated perfusion $(\mathrm{rCBV}>1.75)$, while $17 / 20$ of the HGA group (85\%) showed markedly elevated perfusion.

In analyzing $\mathrm{ADC}$ values, there was a statistically significant difference between the PA group and the HGA group (Table 2 and Fig 2).

The Lip-Lac ${ }^{\text {tu }} / \mathrm{Cr}^{\text {tu }}$ values were significantly higher in the HGA group than in the PA group (Table 2 and Fig 3 ). There was a trend toward higher Lip-Lac ${ }^{\mathrm{tu}} / \mathrm{Lip}-\mathrm{Lac}{ }^{\mathrm{n}}$ ratios in the HGA group compared with the PA group $(P=.06)$. There were no statistically significant differences between the PA and HGA groups in the remaining metabolite ratios (Table 2).

The sensitivity, specificity, positive predictive value, negative predictive value, and the area under the ROC curve of each of the MR imaging-analyzed parameters at respective cutoff values are shown respectively in Tables 3 and 4 .

\section{DISCUSSION}

In an attempt to differentiate PA and HGA, our data showed that lower $\mathrm{rCBV}$ and higher $\mathrm{ADC}$ values favor a diagnosis of $\mathrm{PA}$, while higher Lip-Lac ${ }^{\mathrm{tu}} / \mathrm{Cr}^{\mathrm{tu}}$ ratios plus necrosis favor a diagnosis of HGA.

Although PA is classified as grade I by the WHO because of its benign biologic behavior, this tumor can have contradictory findings on both neuroimaging and histopathology and may be confused with a malignant tumor. ${ }^{1,4}$

PA has been commonly described as a large cystic mass with a mural nodule that shows a marked enhancement. ${ }^{1,5,7}$ Therefore, when a typical solid-cystic tumor is found on MR imaging in a patient younger than 20 years of age, the most probable diagnosis is $\mathrm{PA},{ }^{1,5}$ particularly when it is located in the posterior fossa.

AJNR Am J Neuroradiol 35:1495-502 Aug 2014 www.ajnr.org 

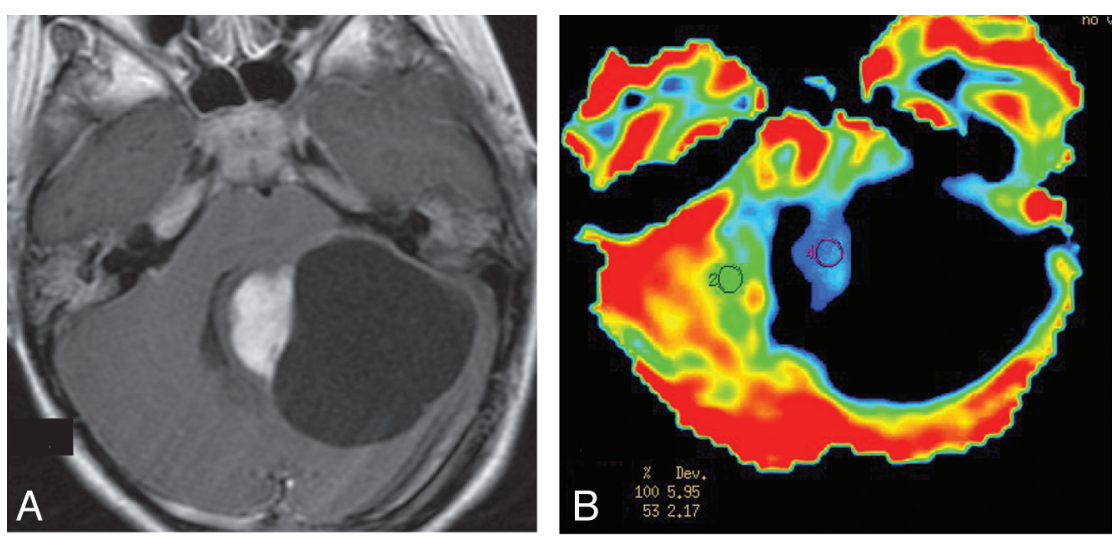

entiate PA and HGA, ${ }^{1,4}$ in which case advanced MR imaging could be crucial in the diagnosis of the lesion.

Several studies have demonstrated that $\mathrm{rCBV}$ has clinical utility in glioma grading. However, most of these studies only included WHO grade II tumors in the low-grade glioma (LGG) group and WHO grades III and IV in the HGG group. ${ }^{10,16,17}$ Most of these studies failed to include PA in the LGG group or even to consider it as a separate cohort. ${ }^{9,10,16,17}$ To our knowledge, there are only a few studies assessing PWI in PAs, all

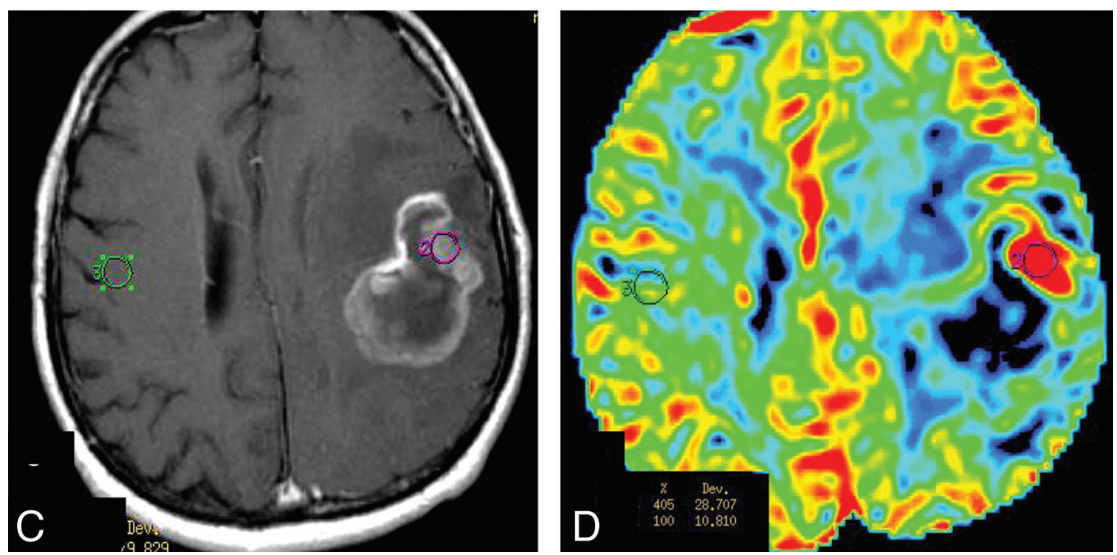

FIG 1. Comparison of rCBV between PA and HGA on PWI. $A$ and $B$, Solid-cystic PA in the left cerebellar hemisphere. $A$, Contrast-enhanced axial T1-weighted imaging demonstrates intense homogeneous enhancement in the solid portion of the tumor and lack of enhancement of the walls of the cystic portion. $B, \mathrm{PWI}$, with an rCBV color overlay map, shows low rCBV on the solid portion of PA. C and D, GBM in the left frontoparietal lobe. C, Contrast-enhanced axial T1weighted imaging demonstrates ring and irregular peripheral enhancement with central necrosis. $D$, PWI with an rCBV color overlay map shows increased rCBV in the solid tumor area. of which are case series or anecdotal case reports in review articles. ${ }^{7,18-21}$

Despite the usual avid enhancement on conventional MR imaging in both PAs and HGAs, the present study found significantly lower rCBV values in PAs compared with HGAs. Some small case series of PAs have also demonstrated low rCBV values $(<1.5)$ in $\mathrm{PA},{ }^{7,12,19}$ while HGAs show high rCBV values in analytical studies (3.64-7.32). 9,10,16,17,22,23 To the best of our knowledge, there are only 2 case reports in review articles that have found high perfusion in PAs. ${ }^{18,21}$

Uematsu et $\mathrm{al}^{12}$ demonstrated in a case series that the mean vascularity index of GBMs is higher than that of PAs, but the mean vascular leakage was lower in GBMs. This finding could be ex-

Conversely, when imaging reveals a single tumor with ring enhancement, suggesting central necrosis, the most probable diagnosis is HGA, specifically GBM. ${ }^{14}$ These MR imaging features were found in $60 \%$ of the HGAs and in only $7 \%$ of the PAs in our series.

However, when the tumor is solid and seems to infiltrate adjacent brain tissue with intense enhancement, as it did in $50 \%$ of the PAs and $37 \%$ of the HGAs in our study, there is less certainty as to whether the tumor is a PA or HGA. This ambiguity can occur even in patients who are younger than 20 years of age, when age favors a diagnosis of $\mathrm{PA},{ }^{1}$ as well as in adult patients, in whom PA is rare. ${ }^{2}$

Advanced MR imaging techniques can help distinguish these entities much better than conventional imaging alone. ${ }^{8,14,15}$ Enhanced accuracy helps in treatment planning and prognostication before surgery. ${ }^{8,14}$ This information is even more important when histopathologic diagnosis cannot be obtained due to contraindications to surgery, such as when there is tumor involvement of eloquent areas (motor, speech, or visual brain areas) or when the biopsy tissue collected is insufficient for diagnosis. ${ }^{11}$ Advanced MR imaging techniques can also help overcome sampling errors in the histopathologic grading of tumor and can sample the entire lesion noninvasively in vivo. ${ }^{10}$ Furthermore, there may be instances when a neuropathologist is unable to definitively differ- plained by the different features of PAs and GBMs on histopathology. In the present study, all patients had pathologic confirmation but no formal qualitative evaluation of the neoplasia vascularity was performed. Uematsu et $\mathrm{al}^{12}$ reported that PAs have sparse vasculature in a wide interstitial space, while GBMs have abundant vascularity in a tight interstitial space. ${ }^{12}$ According to some electron microscopy studies of the gap junctions of the endothelial cells, PAs and HGGs probably have similar blood-brain barrier integrity. ${ }^{24,25}$ Taken together, we may postulate that the decreased rCBV observed in PA tumors strongly suggests a decreased vascularity in this low-grade astrocytoma compared with HGA.

Information on the metabolism of brain tissue may be obtained from proton MR spectroscopy. ${ }^{11,26}$ The ability of MR spectroscopy to predict glioma grade is controversial. ${ }^{11}$ The presence of lipids has been recognized as a marker of myelin breakdown, as well as a potential indicator of necrosis in malignant astrocytomas. ${ }^{27}$ Lactate is considered an end product of the anaerobic glycolytic cycle, and its presence within a tumor often indicates tumor necrosis. ${ }^{10,26}$ The prominent lipid peak, which resonates at 1.3 ppm observed in HGAs, corresponds, mainly, to the methylene group of lipid molecules, whereas in PA, it is mainly a lactate peak. 

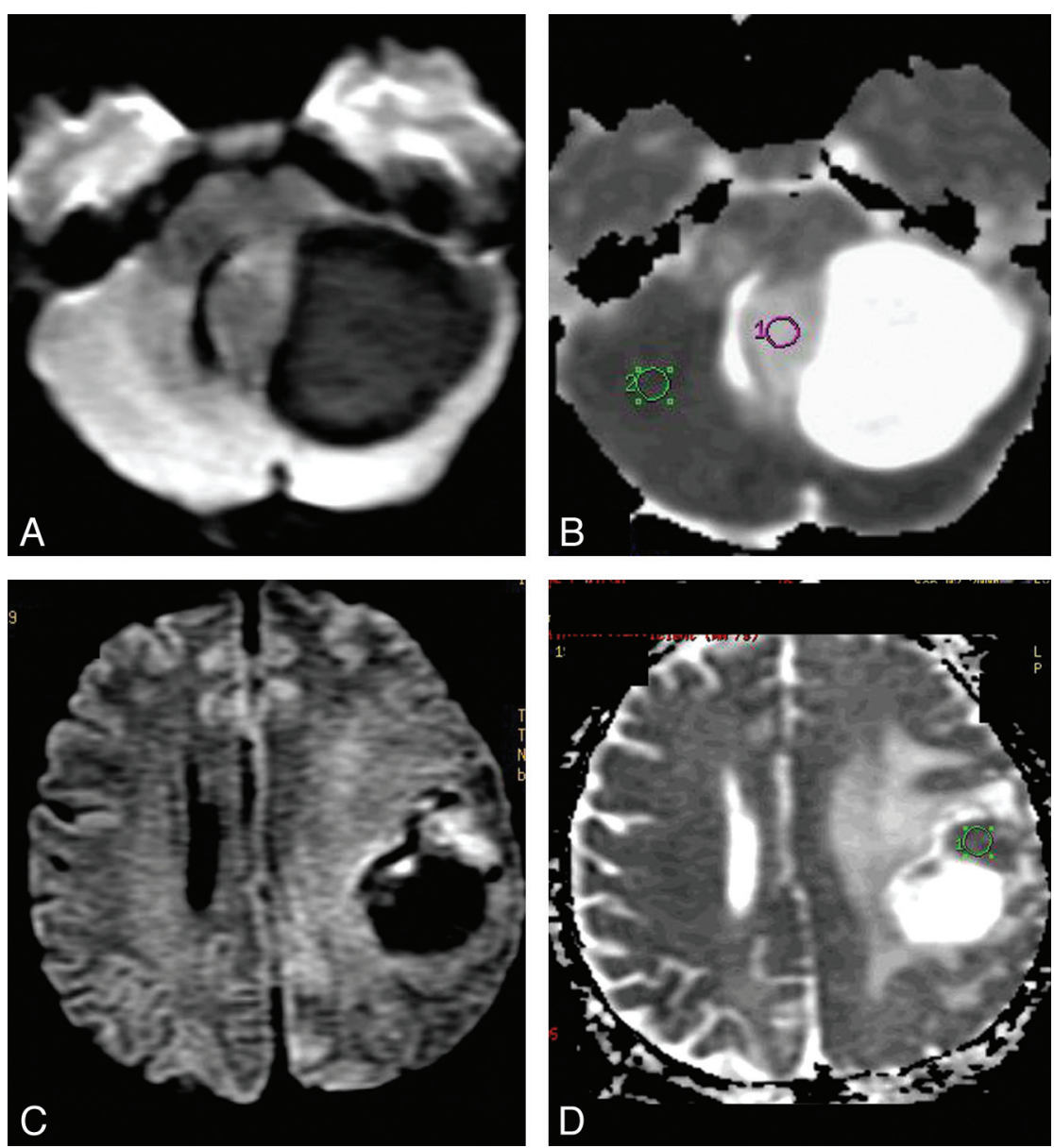

FIG 2. DWI in PA and HGA. $A$ and $B$, Solid-cystic PA in the left cerebellar hemisphere. The solid part of this tumor is hypointense on $\mathrm{DWI}(A)$ and hyperintense on $\mathrm{ADC}$ map $(B)$ in comparison with normal brain parenchyma. $C$ and $D$, The frontoparietal HGA (GBM) shows decreased water diffusion $(C)$ in the more hyperintense solid tumor area on DWI, which is more hypointense $(D)$ on $A D C$ than the normal brain parenchyma and indicates greater cellularity of this GBM than is observed in the PA shown $(A$ and $B)$.

Several studies have indicated that lactate is more likely to be present in HGGs than in LGGs, ${ }^{9,28-31}$ but most of these studies did not include PAs among the LGGs. There have been a few studies assessing the Lip-Lac ${ }^{\text {tu }} / \mathrm{Cr}^{\text {tu }}$ ratio in PAs, but all used long-TE (270 ms) single-voxel spectroscopy. ${ }^{26,32}$ Although necrosis is seldom found in PAs, Hwang et $\mathrm{al}^{26}$ described the presence of lipids and lactate in PAs, despite their benign tumor histology.

In our study, among all metabolite ratios analyzed, only Lip-Lac ${ }^{\text {tu }}$ $\mathrm{Cr}^{\text {tu }}$ values were significantly higher in HGAs than in PAs. There was also a trend toward a higher Lip-Lac ${ }^{\text {tu }} / \mathrm{Lip}_{-\mathrm{Lac}^{\mathrm{n}}}$ ratio in HGAs. The Lip$\mathrm{Lac}^{\mathrm{tu}} / \mathrm{Cr}^{\mathrm{tu}}$ ratio was present and increased in both tumor types, but it was much higher in HGAs, probably due to the presence of tumoral necrosis. Although the presence of Lip-Lac in PAs in the absence of necrosis can be explained by several mechanisms, including mitochondrial dysfunction, ${ }^{26}$ our hypothesis is that it may be attributed to the commonly occurring microscopic cysts on histopathology.

As in previous reports, ${ }^{26,32-35}$ we also found increased $\mathrm{Cho}^{\mathrm{tu}} /$ $\mathrm{NAA}^{\text {tu }}$ and $\mathrm{Cho}^{\text {tu }} / \mathrm{Cr}^{\text {tu }}$ ratios in PAs, with values lower than those in some studies, ${ }^{26,33}$ but higher than those of Sutton et al. ${ }^{32}$ Some authors made the comparison between gliomas without including PAs and found higher $\mathrm{Cho}^{\text {tu }} / \mathrm{NAA}^{\text {tu }}$ and $\mathrm{Cho}^{\text {tu }} / \mathrm{Cr}^{\text {tu }}$ ratios in
HGGs than in non-PA LGGs, ${ }^{9,10,36}$ very likely due to the greater cellularity of malignant tumors. However, our study found the opposite to be true in the comparison between PA, which is an LGG, and HGA, though the difference was not significant. Our finding may be explained by the presence of necrosis within the GBMs, which decreases the number of neoplastic cells and consequently lowers the $\mathrm{Cho}^{\mathrm{tu}} / \mathrm{NAA}^{\text {tu }}$ and $\mathrm{Cho}^{\text {tu }} / \mathrm{Cr}^{\mathrm{tu}}$ ratios $^{11}$ in the HGA group. This process does not occur with the PAs that showed higher values of these ratios.

It has been demonstrated that the MR spectroscopy pattern appears slightly different between PA in adults and in children, though no significant differences have been identified. ${ }^{37}$ Only 5 PAs were investigated with MR spectroscopy in our study, in 4 children (8, $12,12,14$ years of age) and 1 adult (20 years of age). Similar to the previous article, ${ }^{37}$ our study also shows that the normalized $\mathrm{Cr}\left(\mathrm{Cr}^{\mathrm{tu}} / \mathrm{Cr}^{\mathrm{n}}\right)$ was reduced in all PAs. As in the previous article, ${ }^{37}$ the reduced normalized mean $\mathrm{Cr}^{\mathrm{tu}} / \mathrm{Cr}^{\mathrm{n}}$ ratio (0.52) in the $\mathrm{PA}$ pediatric group was higher compared with the PA of the adult patient ratio (0.26). All our cases in the PA pediatric group presented increased values of normalized Cho (Cho ${ }^{\text {tu }}$ $\mathrm{Cho}^{\mathrm{n}}$ ) according to the previous study. ${ }^{37}$ However, unlike the previous study, ${ }^{37}$ in our study, the normalized Cho $\left(\mathrm{Cho}^{\text {tu }} / \mathrm{Cho}^{\mathrm{n}}\right)$ of our 1 adult patient was low (0.97) and lower than the mean of the 1.5 ratio in the pediatric group. It is always difficult to compare the results of studies because usually different equipment (eg, the prior study used 3T MR imaging), different technical parameters of MR spectroscopy (eg, the prior study used $\mathrm{TE}=144 \mathrm{~ms}),{ }^{37}$ and different methodologies are used. The small number of cases of PA in the pediatric and adult groups was another major limitation in both studies.

Castillo et $\mathrm{al}^{38}$ suggest that mIns levels could be a marker for astrocytoma grade, with a trend toward higher mIns levels in lowgrade astrocytomas (including both WHO grade I and II tumors) compared with anaplastic astrocytomas and GBMs. ${ }^{28,38}$ In our study, the means of the $\mathrm{mIns}^{\mathrm{tu}} / \mathrm{Cr}^{\mathrm{tu}}$ and $\mathrm{mIns} \mathrm{s}^{\mathrm{tu}} / \mathrm{mIns}^{\mathrm{n}}$ ratios were also higher in PAs than in HGAs, but the differences were not statistically significant. Schneider et al, ${ }^{39}$ by using a different technique to normalize metabolites (water was used as an internal reference), found that grade II gliomas demonstrated significantly higher levels of mIns than PAs. However, contradictory reports have also demonstrated low mIns levels in PAs. ${ }^{34,40}$

AJNR Am J Neuroradiol 35:1495-502 Aug 2014 www.ajnr.org 

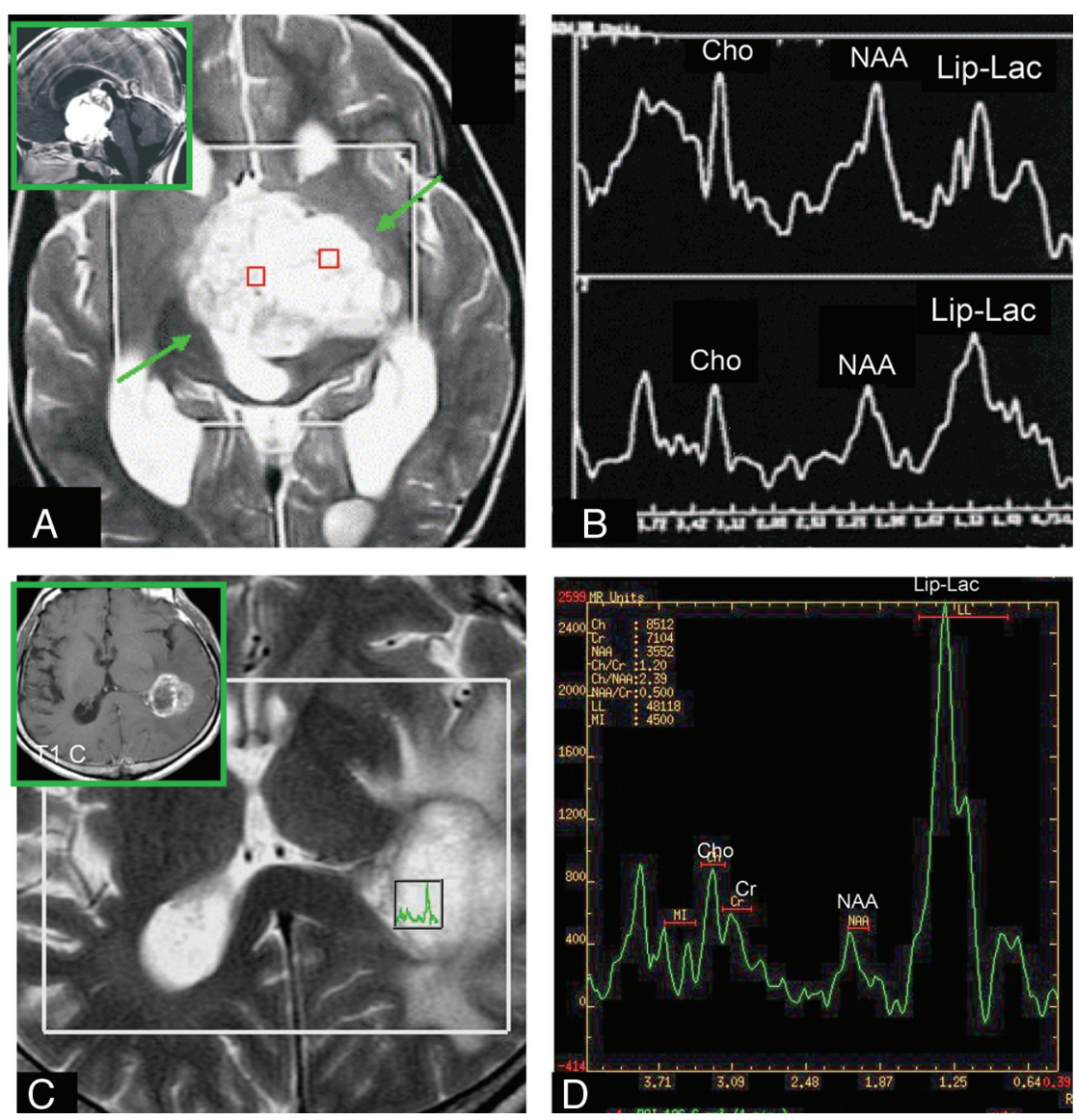

FIG 3. Comparison of the Lip-Lac ${ }^{\mathrm{tu}} / \mathrm{Cr}^{\mathrm{tu}}$ ratio on short-TE multivoxel MR spectroscopy in PA and HGA. $A$ and C, Axial T2-weighted imaging was used for positioning of the regions of interest in the tumor. $A$ and $B$, The solid homogeneous enhancing hypothalamic-chiasmatic PA has a Lip-Lac peak. The ( $C$ and $D)$ necrotic ring-enhancing GBM located on the lateral wall of the left lateral ventricular trigone also has an elevated Lip-Lac peak $(D)$, but the $\mathrm{Lip}-\mathrm{LaC}^{\mathrm{tu}} / \mathrm{Cr}^{\mathrm{tu}}$ ratio in this tumor is higher than that found in the PA (B). $A$ and $B$ reproduced with permission from Aragao et al. ${ }^{47}$

Table 3: Cutoff values, sensitivity, specificity, positive predictive value, negative predictive value, and accuracy of the different studied MRI parameters to identify high-grade (III/IV) astrocytomas (HGA)

\begin{tabular}{lcccccc}
\hline \multicolumn{1}{c}{ Parameter } & Cutoff Value $^{\text {a }}$ & Sensitivity & Specificity & PPV & NPV & Accuracy \\
\hline rCBV & $\geq 1.33$ & $100 \%$ & $67 \%$ & $87 \%$ & $100 \%$ & $90 \%$ \\
ADC & $\leq 1.60$ & $100 \%$ & $54 \%$ & $63 \%$ & $100 \%$ & $72 \%$ \\
Lip-Lac $^{\text {tu }} / C r^{\text {tu }}$ & $\geq 7.06$ & $80 \%$ & $80 \%$ & $94 \%$ & $50 \%$ & $80 \%$ \\
Lip-Lac $^{\text {tu }} /$ Lip-Lac $^{\text {n }}$ & $\geq 2.11$ & $73 \%$ & $80 \%$ & $94 \%$ & $44 \%$ & $76 \%$ \\
\hline
\end{tabular}

Note:-PPV indicates positive predictive value; NPV, negative predictive value.

${ }^{\text {a } H G A}$ group.

Table 4: Areas under the receiver operating characteristic analysis curve for the variables of interest for distinguishing PA from HGA

\begin{tabular}{lccc}
\multicolumn{1}{c}{ Parameters } & Area & $\mathbf{9 5 \%}$ Cl & $\boldsymbol{P}$ Value \\
\hline rCBV & 0.906 & $0.790-1.000$ & .001 \\
ADC & 0.795 & $0.610-0.980$ & .012 \\
Lip-Lac $^{\text {tu }} /$ Cr $^{\text {tu }}$ & 0.830 & $0.626-1.000$ & .025 \\
Lip-Lac $^{\text {tu }} /$ Lip-Lac $^{\text {n }}$ & 0.780 & $0.585-0.975$ & .047 \\
\hline
\end{tabular}

The diffusion of water in brain tissue may vary in magnitude and direction, depending on several factors; ADC measurements can offer quantitative information regarding the restriction of movement of water molecules. ${ }^{9,41}$ In our series, HGAs demonstrated significantly lower minimum ADC values than PAs $(P=$
.01). The difference may be related to the increased cellularity of higher grade tumors, ${ }^{42,43}$ with resultant restriction of water movement in the interstitial space. ${ }^{43,44}$ Although measurements of necrosis could potentially elevate ADC values, ${ }^{9,43}$ we analyzed the minimum ADC of the solid enhancing portion of the lesion, taking care to exclude the necrotic areas frequently seen in highgrade tumors. Other studies, by using a technique similar to ours, have shown similar results ${ }^{9,43,45}$ when comparing HGGs and LGGs. However, these studies did not include PA cases. Most of the reports that have assessed ADC in PAs have compared them with medulloblastomas, ependymomas, or other LGGs. ${ }^{39,46}$

As far as we know, this is the first time that PAs are compared with HGAs by using the described advanced MR imaging techniques together. The methodology used sought to follow that used in histopathology: to seek brain tumor markers that represent the criteria of greater malignancy. The sensitivity, specificity, predictive values, and accuracy for $\mathrm{rCBV}, \mathrm{ADC}$, $\mathrm{Lip}-\mathrm{Lac}^{\mathrm{tu}} / \mathrm{Cr}^{\mathrm{tu}}$, and Lip-Lac ${ }^{\text {tu }} /$ Lip-Lac ${ }^{n}$ thresholds were determined as the optimal cutoff value in differentiating PAs from HGAs.

This study has some limitations. First, because it is a retrospective study, a full complement of imaging was not available for each patient. Therefore, we have a small population of cases, mainly of pilocytic astrocytomas, with advanced MR imaging techniques. Second, we used a short TE of $35 \mathrm{~ms}$. Even though short-TE MR spectroscopy has the advantage of showing the mIns peak and has better sensitivity to the presence of lipids, its disadvantage is that one cannot separate the lipid peak from the lactate peak because both appear at $1.33 \mathrm{ppm}$ above the baseline. Additionally, there is more noise at the baseline in short-TE than in long-TE MR spectroscopy; and because the glutamine-glutamate peak is close to the NAA peak, it is difficult to properly measure the NAA peak when it is low. Finally, choosing a region of interest to evaluate the rCBV leads to another difficulty in cases in which the tumor is in the gray matter because it is unclear whether the high perfusion value is due to the properties of the gray matter or to the tumor itself. Another limitation of our study is the lack of a multiparametric analysis, which would demon- 
strate the relative contribution of all the clinical, conventional MR imaging and advanced findings to the diagnosis of PA versus HGA. We hope that further investigation will be made by prospective studies with a greater number of cases and multiparametric analysis, by using advanced MR imaging techniques to confirm our findings.

\section{CONCLUSIONS}

We have demonstrated that PWI (rCBV) is one of the best MR imaging techniques in differentiating PA from HGA. Lower rCBV and higher ADC values favor a diagnosis of $\mathrm{PA}$, while higher $\mathrm{rCBV}$ values, lower $\mathrm{ADC}$ values, and higher Lip- $\mathrm{Lac}{ }^{\mathrm{tu}} / \mathrm{Cr}^{\mathrm{tu}}$ ratios plus necrosis favor a diagnosis of HGA. Even though the histopathologic and conventional MR imaging features of PA may occasionally suggest a more malignant tumor, patient age and advanced MR imaging findings should be considered together with the pathologic characteristics to establish the final diagnosis in difficult cases. In this way, the findings of our study may contribute to a change in the current clinical approach, especially in difficult cases encountered by radiologists as well as pathologists in differentiating these 2 types of tumors.

Disclosures: Meng Law_UNRELATED: Board Membership: Bayer HealthCare, Consultancy: iCAD Inc, Payment for Lectures (including service on Speakers Bureaus): Toshiba America Medical.

\section{REFERENCES}

1. Koeller KK, Rushing EJ. From the archives of the AFIP: pilocytic astrocytoma: radiologic-pathologic correlation. Radiographics 2004; 24:1693-708

2. Stuer C, Vilz B, Majores M, et al. Frequent recurrence and progression in pilocytic astrocytoma in adults. Cancer 2007;110:2799-808

3. Burkhard C, Di Patre PL, Schuler D, et al. A population-based study of the incidence and survival rates in patients with pilocytic astrocytoma. J Neurosurg 2003;98:1170-74

4. Scheithauer BW, Hawkins C, Tihan T, et al. Pilocytic astrocytoma. In: Louis DN, Ohgaki H, Wiestler OD, et al, eds. WHO Classification of Tumours of Central Nervous System. Lyon, France: IARC; 2007:14-21

5. Lee YY, Van Tassel P, Bruner JM, et al. Juvenile pilocytic astrocytomas: CT and MR characteristics. AJR Am J Roentgenol 1989;152:1263-70

6. Kuroiwa T, Ohta T, Tsutsumi A. Malignant pilocytic astrocytoma in the medulla oblongata: case report. Brain Tumor Pathol 1999; 16:81-85

7. Grand SD, Kremer S, Tropres IM, et al. Perfusion-sensitive MRI of pilocytic astrocytomas: initial results. Neuroradiology 2007;49:545-50

8. Cha S, Knopp EA, Johnson G, et al. Intracranial mass lesions: dynamic contrast-enhanced susceptibility-weighted echo-planar perfusion MR imaging. Radiology 2002;223:11-29

9. Yang D, Korogi Y, Sugahara T, et al. Cerebral gliomas: prospective comparison of multivoxel $2 \mathrm{D}$ chemical-shift imaging proton MR spectroscopy, echoplanar perfusion and diffusion-weighted MRI. Neuroradiology 2002;44:656-66

10. Law M, Yang S, Wang H, et al. Glioma grading: sensitivity, specificity, and predictive values of perfusion MR imaging and proton MR spectroscopic imaging compared with conventional MR imaging. AJNR Am J Neuroradiol 2003;24:1989-98

11. Aragão Mde F, Otaduy MC, Melo RV, et al. Multivoxel spectroscopy with short echo time: choline/ $\mathrm{N}$-acetyl-aspartate ratio and the grading of cerebral astrocytomas [in Portuguese]. Arq Neuropsiquiatr 2007;65:286-94
12. Uematsu H, Maeda M, Sadato N, et al. Measurement of the vascularity and vascular leakage of gliomas by double-echo dynamic magnetic resonance imaging: a preliminary study. Invest Radiol 2002;37:571-76

13. Louis DN, Reifenberg G, Brat DJ, Ellison DW. Tumours: introduction and neuroepithelial tumors. In Love S, Louis DN, Ellison DW, eds. Greenfield's Neuropathology. London, UK: Edward Arnold Publishers; 2008:1821-2000.

14. Nelson SJ, Cha S. Imaging glioblastoma multiforme. Cancer J 2003;9:134-45

15. Martin AJ, Liu H, Hall WA, et al. Preliminary assessment of turbo spectroscopic imaging for targeting in brain biopsy. AJNR Am J Neuroradiol 2001;22:959-68

16. Sugahara T, Korogi Y, Kochi M, et al. Correlation of MR imagingdetermined cerebral blood volume maps with histologic and angiographic determination of vascularity of gliomas. AJR Am J Roentgenol 1998;171:1479-86

17. Aronen HJ, Gazit IE, Louis DN, et al. Cerebral blood volume maps of gliomas: comparison with tumor grade and histologic findings. $\mathrm{Ra}$ diology 1994;191:41-51

18. Ball WS Jr, Holland SK. Perfusion imaging in the pediatric patient. Magn Reson Imaging Clin N Am 2001;9:207-30, ix

19. Maeda M, Itoh S, Kimura H, et al. Tumor vascularity in the brain: evaluation with dynamic susceptibility-contrast MR imaging. $R a$ diology 1993;189:233-38

20. Uematsu H, Maeda M. Double-echo perfusion-weighted MR imaging: basic concepts and application in brain tumors for the assessment of tumor blood volume and vascular permeability. Eur Radiol 2006;16:180-86

21. Cha S. Dynamic susceptibility-weighted contrast-enhanced perfusion MR imaging in pediatric patients. Neuroimaging Clin $\mathrm{N} \mathrm{Am}$ 2006;16:137-47, ix

22. Shin $\mathrm{JH}$, Lee $\mathrm{HK}$, Kwun $\mathrm{BD}$, et al. Using relative cerebral blood flow and volume to evaluate the histopathologic grade of cerebral gliomas: preliminary results. AJR Am J Roentgenol 2002;179:783-89

23. Knopp EA, Cha S, Johnson G, et al. Glial neoplasms: dynamic contrast-enhanced $\mathrm{T} 22^{*}$-weighted $\mathrm{MR}$ imaging. Radiology 1999;211:791-98

24. Nir I, Levanon D, Iosilevsky G. Permeability of blood vessels in experimental gliomas: uptake of $99 \mathrm{mTc}$-glucoheptonate and alteration in blood-brain barrier as determined by cytochemistry and electron microscopy. Neurosurgery 1989;25:523-31

25. Long DM. Capillary ultrastructure and the blood-brain barrier in human malignant brain tumors. J Neurosurg 1970;32:127-44

26. Hwang JH, Egnaczyk GF, Ballard E, et al. Proton MR spectroscopic characteristics of pediatric pilocytic astrocytomas. AJNR Am J Neuroradiol 1998;19:535-40

27. Kuesel AC, Briere KM, Halliday WC, et al. Mobile lipid accumulation in necrotic tissue of high grade astrocytomas. Anticancer Res 1996;16:1485-89

28. Howe FA, Barton SJ, Cudlip SA, et al. Metabolic profiles of human brain tumors using quantitative in vivo $1 \mathrm{H}$ magnetic resonance spectroscopy. Magn Reson Med 2003;49:223-32

29. Di Costanzo A, Scarabino T, Trojsi F, et al. Multiparametric 3T MR approach to the assessment of cerebral gliomas: tumor extent and malignancy. Neuroradiology 2006;48:622-31

30. Kaminogo M, Ishimaru H, Morikawa M, et al. Diagnostic potential of short echo time MR spectroscopy of gliomas with single-voxel and point-resolved spatially localised proton spectroscopy of brain. Neuroradiology 2001;43:353-63

31. Tzika AA, Zarifi MK, Goumnerova L, et al. Neuroimaging in pediatric brain tumors: Gd-DTPA-enhanced, hemodynamic, and diffusion MR imaging compared with MR spectroscopic imaging. AJNR Am J Neuroradiol 2002;23:322-33

32. Sutton LN, Wang Z, Gusnard D, et al. Proton magnetic resonance spectroscopy of pediatric brain tumors. Neurosurgery 1992;31:195-202

33. Sutton LN, Wehrli SL, Gennarelli L, et al. High-resolution 1H-mag- 
netic resonance spectroscopy of pediatric posterior fossa tumors in vitro. J Neurosurg 1994;81:443-48

34. Panigrahy A, Krieger MD, Gonzalez-Gomez I, et al. Quantitative short echo time 1H-MR spectroscopy of untreated pediatric brain tumors: preoperative diagnosis and characterization. AJNR Am J Neuroradiol 2006;27:560-72

35. Lazareff JA, Olmstead C, Bockhorst KH, et al. Proton magnetic resonance spectroscopic imaging of pediatric low-grade astrocytomas. Childs Nerv Syst 1996;12:130-35

36. Fulham MJ, Bizzi A, Dietz MJ, et al. Mapping of brain tumor metabolites with proton MR spectroscopic imaging: clinical relevance. Radiology 1992;185:675-86

37. Porto L, Kieslich M, Franz K, et al. Spectroscopy of untreated pilocytic astrocytomas: do children and adults share some metabolic features in addition to their morphologic similarities? Childs Nerv Syst 2010;26:801-06

38. Castillo M, Smith JK, Kwock L. Correlation of myo-inositol levels and grading of cerebral astrocytomas. AJNR Am J Neuroradiol 2000;21:1645-49

39. Schneider JF, Confort-Gouny S, Viola A, et al. Multiparametric differentiation of posterior fossa tumors in children using diffusionweighted imaging and short echo-time 1H-MR spectroscopy. $J$ Magn Reson Imaging 2007;26:1390-98
40. Schneider JF, Viola A, Confort-Gouny S, et al. Infratentorial pediatric brain tumors: the value of new imaging modalities. J Neuroradiol 2007;34:49-58

41. Tanner JE. Intracellular diffusion of water. Arch Biochem Biophys $1983 ; 224: 416-28$

42. Cha S. Update on brain tumor imaging: from anatomy to physiology. AJNR Am J Neuroradiol 2006;27:475-87

43. Sugahara T, Korogi $\mathrm{Y}$, Kochi M, et al. Usefulness of diffusionweighted MRI with echo-planar technique in the evaluation of cellularity in gliomas. J Magn Reson Imaging 1999;9:53-60

44. Le Bihan D, Breton E, Lallemand D, et al. MR imaging of intravoxel incoherent motions: application to diffusion and perfusion in neurologic disorders. Radiology 1986;161:401-07

45. Murakami R, Hirait T, Kitajima M, et al. Magnetic resonance imaging of pilocytic astrocytomas: usefulness of the minimum apparent diffusion coefficient (ADC) value for differentiation from highgrade gliomas. Acta Radiol 2008;49:462-67

46. Yamasaki F, Kurisu K, Satoh K, et al. Apparent diffusion coefficient of human brain tumors at MR imaging. Radiology 2005;235:985-91

47. Aragao MF, Soares ML, Holanda GR. Tumores supratentoriais. In: Silva CI, D’Ippolito G, Rocha AJ, et al, eds. Série Colégio Brasileiro de Radiologia e Diagnóstico por Imagem- Encéfalo. Rio de Janeiro, Brazil: Elsevier; 2012:292 